\title{
SWIMMERS' SHOULDER SIDE ASYMMETRY AT REST AND AFTER AEROBIC LOAD
}

original paper

( $)$ Wroclaw University of Health and Sport Sciences

DOI: https://doi.org/10.5114/hm.2022.107980

\author{
LIGA IRMANE ${ }^{1,2}$, INESE PONTAGA ${ }^{1 \oplus}$, IMANTS UPITIS $^{2}$, JELENA SOLOVJOVA $^{2}$ \\ ${ }^{1}$ Department of Anatomy, Physiology, Biochemistry, Biomechanics, Hygiene and Informatics, Latvian Academy of Sport Education, \\ Riga, Latvia \\ ${ }^{2}$ Department of Track and Field Athletics and Swimming, Latvian Academy of Sport Education, Riga, Latvia
}

\begin{abstract}
Purpose. The aim of our study was to evaluate shoulder joint flexibility, shoulder muscle peak isometric force, and submaximal force differentiation ability after warm-up exercises, as well as to compare the peak isometric force and submaximal force differentiation ability before and after a $4.5-\mathrm{km}$ free-style swimming trial in swimmers and triathletes.

Methods. Overall, 15 qualified young male swimmers, 15 triathletes, and 14 controls participated. Their shoulder active range of motion (ROM) in internal rotation (IR), external rotation, flexion, extension, abduction were measured. The peak force and the ability to reproduce the submaximal forces of IR and extension muscles were compared before and after 4.5-km free-style swimming in aerobic regimen.

Results. Swimmers and triathletes presented larger ROM in flexion and abduction in both arms, and in IR in the non-dominant shoulder than controls. IR and extension muscle isometric peak forces were higher in swimmers and triathletes compared with controls. Strength side asymmetry was not observed in any group. Only triathletes' IR force was higher in the dominant than in the non-dominant shoulder. The submaximal force reproduction error did not differ among the groups. The peak forces and submaximal force reproduction errors did not change after the 4.5-km swim but caused IR and extension muscles peak force side asymmetry, with stronger muscles in the dominant shoulder.

Conclusions. Free-style swimming at $4.5 \mathrm{~km}$ in aerobic regimen induced shoulder IR and extension muscle peak force side asymmetry without any decrease of their absolute values or significant worsening in the submaximal force reproduction error.
\end{abstract}

Key words: strength, flexibility, proprioception, side asymmetry, swimmers, triathletes

\section{Introduction}

The shoulders and upper extremities represented nearly $90 \%$ of the propulsive power for all main swimming strokes [1]. Shoulder adduction, internal rotation (IR), and extension movements were relevant and highly reproduced in the swimming technique during the propulsive phases of different strokes [2,3]. Adult (23.1 \pm 3.5 years old) elite swimmers had higher maximal isometric strength of the shoulder flexion, extension, abduction, and adduction muscles, and larger lean mass of both arms measured by using dual-energy X-ray absorptiometry in comparison with recreational adult swimmers aged $20.8 \pm 2.1$ years [4]. The absolute and relative shoulder IR isometric strength increased in 14-18-year-old elite male swimmers during a 3-year period of training [5]. Shoulder extension torque and velocity while swimming the crawl stroke for $25 \mathrm{~m}$ were correlated with shoulder extension muscle strength [6]. All these investigations proved that the training process induced an increase in strength of the main muscles involved in the swimming technique (adduction, IR, and extension muscles).

A large range of motion (ROM) in the glenohumeral joint gave advantage by allowing a swimmer to achieve a body position that reduced drag, and thus a greater stroke length, correlated directly with speed [7]. Unfortunately, a high flexibility of this joint was the reason for shoulder laxity with increased translation of the humeral head; generalized laxity could be present in

Correspondence address: Inese Pontaga, Department of Anatomy, Physiology, Biochemistry, Biomechanics, Hygiene and Informatics, Latvian Academy of Sport Education, Riga, Brivibas Street 333, LV 1006, Latvia, e-mail: Inese.Pontaga@lspa.lv, https://orcid.org/0000-0001-6659-839X

Received: October 4, 2020

Accepted for publication: March 14, 2021

Citation: Irmane L, Pontaga I, Upitis I, Jelena Solovjova. Swimmers' shoulder side asymmetry at rest and after aerobic load. Hum Mov. 2022;23(3):130-139; doi: https://doi.org/10.5114/hm.2022.107980. 
up to $62 \%$ of competitive swimmers, but a moderate degree of multidirectional instability could be observed in the majority of athletes [8]. A high prevalence of shoulder rotator cuff and biceps muscle tendinopathy was reported in Olympic level swimmers by ultrasonography. It was more common in athletes with a positive sulcus sign, which suggests a role of shoulder laxity in the development of this pathology [9]. The main structure providing the shoulder joint stability during movements consists of the rotator cuff muscles [8]. Therefore, muscle forces are critical for maintaining stability, proper motion, and painless function.

The absolute and relative shoulder IR isometric strength increased simultaneously with external rotation (ER) strength decrease during a 3-year period of regular training in 14-18-year-old male elite swimmers [5], which caused non-balanced action of the shoulder girdle muscles.

Shoulder peak torques and total work detected by isokinetic shoulder rotation strength measurement were significantly higher in competitive adolescent swimmers than in controls at the movement velocities of $60 \%$ s and $180 \%$ s [10]. Front crawl swimmers had lower dominant shoulder ER/IR peak torques ratio $(0.52)$ than sedentary subjects $(0.75)$ at the velocity of $60 \%$ [11], which confirmed previous results [10]. Shoulder IR peak torque increase during a training season in adolescent swimmers was determined [12].

Even though swimming is a symmetrical sport, intensive front crawl swimming involves asymmetry, with a stronger dominant shoulder (the peak torque equalled $61.2 \pm 10.2 \mathrm{~N} \cdot \mathrm{m}$ for the dominant and $52.8 \pm 9.8$ $\mathrm{N} \cdot \mathrm{m}$ for the non-dominant shoulder) [11]. Asymmetries in the shoulder roll that reflected breathing preference were observed, and although the athletes swam without breathing, they persistently rolled more to the side at which they preferred to breathe [13]. Swimmers might apply larger propulsive forces when pulling with the dominant arm [14]. The catch and pull phases of the front crawl stroke were longer for the dominant than for the non-dominant arm [15]. The discrepancy was greater among sprinters than in middle- or longdistance swimmers as the former seek to apply larger forces with each stroke [15]. The underwater swimming stroke phases of the right arm were longer in competitive male swimmers, probably because they were right-handed [16].

Swimmers at the elite level swam up to 4 hours per day, performing approximately 1.5 million stroke cycles per arm per year, and the average competitive swimmer swam approximately 60,000-80,000 m per week [1]. These repetitive and high-intensity movements might result in shoulder muscle fatigue, leading to a worsening of the swimming technique, proprioceptive sense acuity, and swimming performance. The short-term deteriorating effect of fatigue on adult competitive male swimmers' coordination and performance caused by swimming a 200-m maximum in front crawl in a 25-m swimming pool and evaluated by video analysis was confirmed: after this trial, the swimming speed decreased by $13.8 \%$, stroke frequency by $5.1 \%$, stroke length by $6.9 \%$, and angular velocity of the arm by $13.3 \%$ (the load was anaerobic because blood lactate concentration after this swim trial was high: $11.12 \pm$ $1.65 \mathrm{mmol} / 1$ ) [16]. A typical swimming session lasting 2 hours caused significant shoulder ER ROM decrease by $3.4^{\circ}$ without significant changes in the IR range, joint position sense error increase by $2.0^{\circ}$, and pectoralis minor muscle length reduction by $0.7 \mathrm{~cm}$ in male and female elite swimmers [17]. The precision of the shoulder ER position sense worsened in swimmers owing to fatigue after swimming an $8 \times 100 \mathrm{~m}$ distance with 2-minute resting intervals [18]. Shoulder IR and ER force reproduction tests were found to be highly reliable, with interclass correlation coefficients of 0.849 in patients with rotator cuff tendinopathy and 0.909 in healthy subjects; the errors of ER were significantly larger than those of IR, and relative IR and ER force reproduction errors were similar in both groups of participants [19]. Prolonged swimming might deteriorate shoulder muscle exerted contraction force differentiation. This might worsen the accurate replication of swimming movement patterns, crucial to performance and injury prevention because a proper swimming technique significantly reduces the energy expenditure and prevents fast fatigue development in the shoulder muscles. We did not find scientific reports on force reproduction error measured in the shoulders of athletes trained in sports with overhead arm motions.

The aim of our study was to evaluate shoulder joint flexibility, shoulder muscle peak isometric force, and submaximal force differentiation ability after warmup exercises, as well as to compare the peak isometric force and submaximal force differentiation ability before and after a $4.5-\mathrm{km}$ free-style swimming trial in swimmers and triathletes.

We hypothesized that swimming athletes would exhibit larger shoulder ROM in IR-ER, flexion-extension, and abduction, as well as higher peak isometric force and smaller force reproduction error of IR and extension muscles in comparison with the controls; moreover, swimming $4.5 \mathrm{~km}$ in free style in aerobic regimen could diminish the peak isometric force and worsen the submaximal force differentiation ability. 


\section{Material and methods}

A total of 15 qualified male swimmers, 15 triathletes (swimming, cycling, and running), and 14 participants in the control group (trained in football, running, or folk dancing, i.e. sports without overhead movements of arms) participated in the study.

The criteria for inclusion were as follows: all swimmers and triathletes trained at least 5 times per week and competed regularly at the national level and in international championships, their training experience in swimming ranged 7-12 years (the mean training experience equalled $9.6 \pm 2.2$ years in swimmers and $10.1 \pm 1.3$ years in triathletes), the mean duration of training sessions of swimming was $10.4 \pm 1.5$ hours per week in swimmers and $8.8 \pm 0.7$ hours per week in triathletes.

The exclusion criteria involved previous surgery or injury of a shoulder joint, or shoulder joint painfulness during the investigation.

The triathletes were significantly older in comparison with the other 2 groups of participants (Table 1). They all were former swimmers trained in the same swimming school as the qualified swimmers.

The measurements were performed in a transitional phase of the training cycle, 2-3 days after a national level competition.

The athletes' height was assessed by using an Ultrasound Height Measuring Unit MZ10020 (ADE, Hamburg, Germany). Body mass was evaluated in participants wearing briefs, with a Body Composition Analyser BC-418 (Tanita Corporation, Tokyo, Japan). The arm length was measured with a tape from the acromion process to the end of the middle finger of the hand. The mean anthropometric characteristics did not differ significantly among the 3 groups of participants (Table 1).

Before the shoulder flexibility and strength measurements, the participants performed general warm-up exercises, local warm-up, and active stretching for shoulders and arms for 15 minutes.

The active shoulder ROM was tested in IR-ER, flexion-extension, and abduction by using Polaroid photography, which had demonstrated better interobserver reliability than goniometric ROM testing, especially in less experienced observers [20]. Markers were placed on the anatomical landmarks: posterolateral acromion process, lateral epicondyle and olecranon process of the humeral bone, ulnar styloid process. The participants were placed in a standard position. They were asked to perform certain active motions as far as possible [21]. A Polaroid photograph was taken of the movement at the end of ROM. The camera was centred in the axis of rotation of the shoulder joint, which was detected, and the movement was performed in the plane of the camera to minimize the parallax error. Lines were drawn on each photograph to connect the markers placed on the anatomical landmarks. Then, the angles formed by these lines were measured by using a protractor with $1^{\circ}$ increments. One bilateral measurement of each of the following active motions was recorded on a photograph.

Shoulder IR-ER ranges were measured with the participants in supine position with the shoulder abducted to $90^{\circ}$, elbow flexed to $90^{\circ}$, and forearm in neutral pronation/supination position. The ER-IR angles were measured between the line formed by the olecranon process and ulnar styloid process and the vertical line which goes through the olecranon process perpendicularly to the horizontal plane in maximal active ER and IR positions of ROM. Assistance was given to keep the shoulder on the table during the IR motion, preventing compensatory use of the shoulder protractor muscles.

Active shoulder flexion was tested with the participants in supine position, with the palm of the hand facing upward and the elbow extended, with the arm over the side of the table. Active shoulder extension was investigated in supine position, with the palm of the hand facing downward and the elbow extended, with the arm over the side of the table. The shoulder flexion-extension angles were measured between the line formed by the acromion process, lateral epicondyle of the humeral bone, and ulnar styloid process and the horizontal line.

Table 1. Comparison of the mean anthropometric characteristics $( \pm S D)$ in qualified swimmers, triathletes, and control group participants

\begin{tabular}{lccccc}
\hline Participants & $\begin{array}{c}\text { Mean age } \\
\text { (years) }\end{array}$ & $\begin{array}{c}\text { Mean height } \\
(\mathrm{cm})\end{array}$ & $\begin{array}{c}\text { Mean body mass } \\
(\mathrm{kg})\end{array}$ & $\begin{array}{c}\text { Mean body mass } \\
\text { index }\left(\mathrm{kg} / \mathrm{m}^{2}\right)\end{array}$ & $\begin{array}{c}\text { Mean arm length } \\
(\mathrm{cm})\end{array}$ \\
\hline Swimmers & $16.9 \pm 1.0$ & $181.6 \pm 4.7$ & $74.3 \pm 8.6$ & $22.5 \pm 2.3$ & $80.9 \pm 3.7$ \\
Triathletes & $20.1^{*} \pm 0.74$ & $183.1 \pm 4.7$ & $76.4 \pm 8.8$ & $22.8 \pm 2.0$ & $82.1 \pm 3.7$ \\
Control group & $17.6 \pm 1.1$ & $179.1 \pm 6.1$ & $73.6 \pm 10.2$ & $22.9 \pm 2.4$ & $79.9 \pm 3.1$ \\
\hline
\end{tabular}

${ }^{*} p<0.0001$ 
Active shoulder abduction was assessed with the participants standing in a vertical position, with the elbow extended and the palm facing to the front. The end point was determined by the participant's maximal active range, precluding observable substitution. The shoulder abduction angles were measured between the line formed by the acromion process, lateral epicondyle of the humeral bone and ulnar styloid process and the vertical line which went through the acromion process perpendicularly to the horizontal plane.

The peak isometric shoulder muscle force was determined by using a hand-held dynamometer (MicroFET2 Wireless; Hoggan Health Industries, West Jordan, USA) in newtons (N), with methods elaborated by Awatani et al. [22-24]. The strength measurements were performed in a prone position of the participants. The arm muscle extension force was evaluated with the shoulder positioned in maximum abduction, elbow extended, and forearm in a neutral position. The shoulder muscle IR force was assessed with the shoulder positioned in $90^{\circ}$ of abduction and $90^{\circ}$ of ER, elbow in $90^{\circ}$ of flexion, and forearm in a neutral position. Each participant performed 3 repetitions of the peak shoulder extension and IR force production, pushing the dynamometer against the floor with as much force as possible continuously for 2 seconds with each arm; a timed rest break of 60 seconds was given between the force measurement trials. The best repetition (highest force) of shoulder extension and IR muscle developed force measurement in each shoulder was considered. The order of the isometric force testing in the dominant and non-dominant shoulder was randomized.

Each participant performed 3 pairs of submaximal force exertion trials. They had to reproduce the same force (the same effort of the muscles) in approximately $50 \%$ of the maximal voluntary contraction continuously for 2 seconds twice, with a 30 -second rest break between the 2 measurements in each trial. A timed rest break between the 3 different trials was 60 seconds. The mean force repetition error of the 3 measurement trials was calculated.

Then, the swimmers and triathletes swam a distance of $4.5 \mathrm{~km}$ in free style in aerobic regimen. The load intensity was determined by heart rate established after swimming of each $500 \mathrm{~m}$ in the $25-\mathrm{m}$ long swimming pool. The heart rate of the participants varied between 138 and 164 beats per minute.

The peak isometric force and submaximal force reproduction error measurements were repeated immediately after this trial of swimming.

The data on height, body mass, and arm length were normally distributed in the 3 groups of participants; shoulder active ROM, maximal shoulder muscle force, and error of the submaximal force repetitions were also normally distributed in both arms. The data distribution was established from the values of the skewness and kurtosis, and skewness and kurtosis $t$-test analysis. The mean values and standard deviations were calculated for all characteristics. A $t$-test for paired samples was employed to determine differences between the mean characteristics of the dominant and non-dominant arms and in the same arms before and after the swimming trial. To compare swimmers, athletes trained in triathlon, and control group participants, a $t$-test for unequal samples was used. The differences were considered significant at $p<0.05$. Microsoft Excel 2010 was applied to perform all statistical procedures.

\section{Ethical approval}

The research related to human use has complied with all the relevant national regulations and institutional policies, has followed the tenets of the Declaration of Helsinki, and has been approved by the Ethics Committee of the Latvian Academy of Sport Education (Meeting Protocol No. 7, decision No. 17/47813 of February 28,2019 ).

\section{Informed consent}

Informed consent has been obtained from all individuals included in this study or from their legal guardians.

\section{Results}

No significant side asymmetry was observed in shoulder ROM between the dominant and non-dominant arm in the shoulder ER-IR, flexion-extension, or abduction among the swimmers, triathletes, and controls $(p>0.450)$ (Table 2). The difference between the shoulder ROMs in swimmers and athletes trained in triathlon for ER-IR, flexion-extension, and abduction was not significant in the dominant or non-dominant arm $(p>0.530)$. Our swimmers and triathletes had significantly larger shoulder ROM in comparison with the controls in both arms in flexion and abduction $(p<0.0001)$. The swimmers and triathletes had significantly larger shoulder IR ROM than the controls in the non-dominant arm $(p<0.03)$.

Shoulder IR and extension muscle peak force did not differ significantly in the dominant and non-dominant arm among swimmers and participants from the control group $(p>0.095)$. A statistically significant IR muscle peak force side asymmetry was determined only in triathletes: IR muscles of the dominant arm 


\section{HUMAN MOVEMENT}

L. Irmane, I. Pontaga, I. Upitis, J. Solovjova, Swimmers' shoulder side asymmetry

Table 2. Comparison of the mean range of motion $( \pm S D)$ in qualified swimmers, triathletes, and controls

\begin{tabular}{lccccc}
\hline Participants, arm & External rotation & Internal rotation & Flexion & Extension & Abduction \\
\hline Swimmers, D & $97 \pm 10^{\circ}$ & $82 \pm 12^{\circ}$ & $194^{* *} \pm 12^{\circ}$ & $63 \pm 9^{\circ}$ & $195^{* *} \pm 5^{\circ}$ \\
Controls, D & $88 \pm 12^{\circ}$ & $79 \pm 15^{\circ}$ & $166 \pm 8^{\circ}$ & $62 \pm 12^{\circ}$ & $170 \pm 4^{\circ}$ \\
Swimmers, N & $95 \pm 11^{\circ}$ & $84^{*} \pm 11^{\circ}$ & $195^{* *} \pm 12^{\circ}$ & $64 \pm 8^{\circ}$ & $195^{* *} \pm 6^{\circ}$ \\
Controls, N & $87 \pm 13^{\circ}$ & $71 \pm 17^{\circ}$ & $165 \pm 7^{\circ}$ & $63 \pm 12^{\circ}$ & $170 \pm 6^{\circ}$ \\
Triathletes, D & $96 \pm 10^{\circ}$ & $83 \pm 11^{\circ}$ & $193^{* *} \pm 12^{\circ}$ & $64 \pm 7^{\circ}$ & $194^{* *} \pm 5^{\circ}$ \\
Controls, D & $88 \pm 12^{\circ}$ & $79 \pm 15^{\circ}$ & $166 \pm 8^{\circ}$ & $62 \pm 12^{\circ}$ & $170 \pm 4^{\circ}$ \\
Triathletes, N & $95 \pm 10^{\circ}$ & $85^{*} \pm 11^{\circ}$ & $195^{* *} \pm 11^{\circ}$ & $65 \pm 8^{\circ}$ & $195^{* *} \pm 6^{\circ}$ \\
Controls, N & $87 \pm 13^{\circ}$ & $71 \pm 17^{\circ}$ & $165 \pm 7^{\circ}$ & $63 \pm 12^{\circ}$ & $170 \pm 6^{\circ}$ \\
\hline
\end{tabular}

$\mathrm{D}$ - dominant arm, $\mathrm{N}$ - non-dominant arm

${ }^{*} p<0.05,{ }^{* *} p<0.0001$

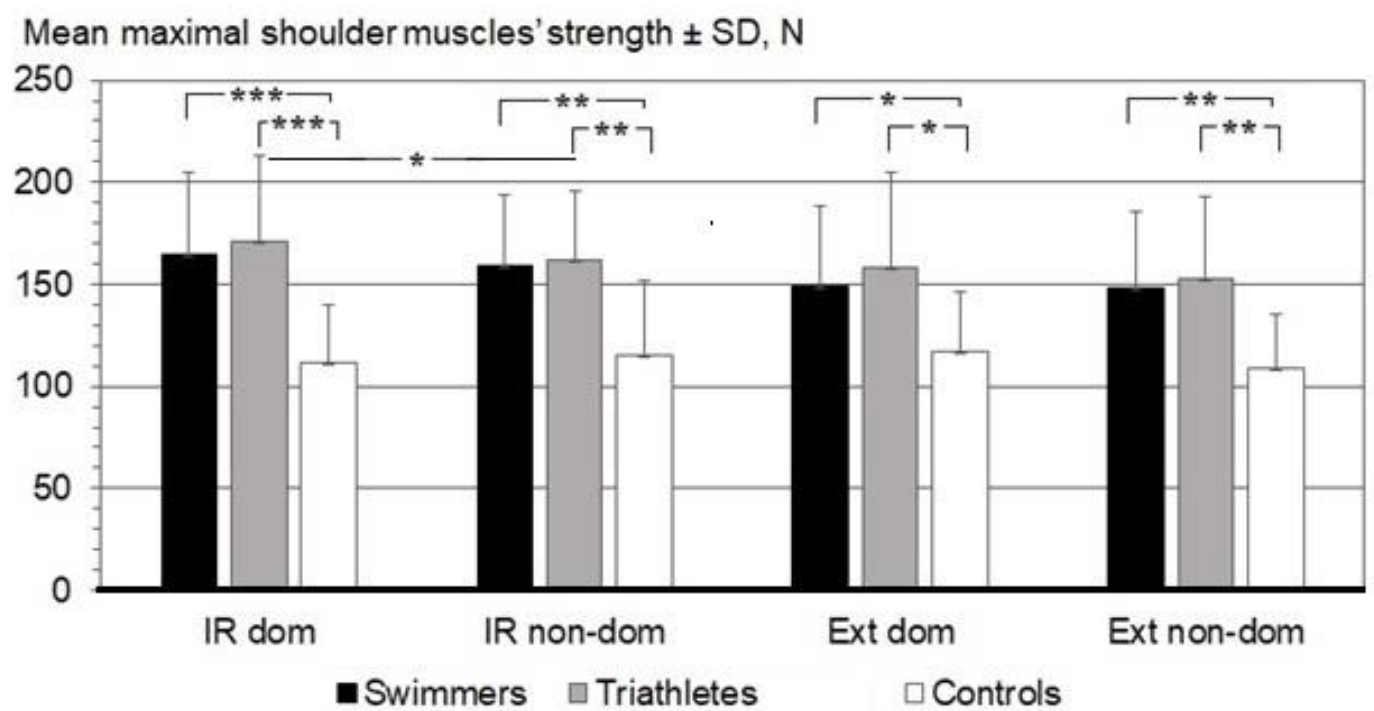

IR - internal rotation, dom - dominant arm, non-dom - non-dominant arm, Ext - extension ${ }^{*} p<0.02,{ }^{* *} p<0.003,{ }^{* * *} p<0.0003$

Figure 1. Comparison of the mean peak force of shoulder muscles in the dominant and non-dominant arm of swimmers, triathletes, and control group participants



Figure 2. The mean shoulder internal rotation and extension muscle submaximal force repetition error in the dominant and non-dominant arm of swimmers, triathletes, and control group participants 
Table 3. Comparison of the shoulder muscle mean peak force $(\mathrm{N} ; \pm S D)$ in swimmers and triathletes before and after swimming $4.5 \mathrm{~km}$ in free style in aerobic regimen

\begin{tabular}{lccrr}
\hline & \multicolumn{4}{c}{ Group of muscles } \\
\cline { 2 - 5 } Participants & \multicolumn{2}{c}{ Shoulder internal rotation muscles } & \multicolumn{2}{c}{ Shoulder extension muscles } \\
\cline { 2 - 5 } & Dominant arm & Non-dominant arm & Dominant arm & Non-dominant arm \\
\hline Swimmers, after warm-up & $165 \pm 40$ & $159 \pm 35$ & $149 \pm 39$ & $148 \pm 38$ \\
Swimmers, after swimming & $180 \pm 49$ & $167^{*} \pm 40$ & $161 \pm 41$ & $144^{* *} \pm 38$ \\
Triathletes, after warm-up & $171 \pm 42$ & $162^{*} \pm 34$ & $158 \pm 47$ & $153 \pm 40$ \\
Triathletes, after swimming & $188 \pm 53$ & $173^{*} \pm 40$ & $169 \pm 46$ & $153^{* * *} \pm 44$ \\
\hline
\end{tabular}

${ }^{*} p<0.05,{ }^{*} p<0.001,{ }^{* *} p<0.0001$

exerted significantly larger peak force than IR muscles of the non-dominant arm ( $p=0.0336)$ (Figure 1). The mean peak force values of the shoulder IR and extension muscles did not differ significantly in swimmers and triathletes $(p>0.574)$, but were significantly higher in both these groups in comparison with the controls $(p<0.02)$.

The shoulder IR and extension muscle mean submaximal force reproduction error did not differ significantly in the dominant and non-dominant arm among swimmers, triathletes, and controls $(p>0.079)$. The mean shoulder muscle submaximal force reproduction errors of shoulder IR and extension muscles did not differ significantly among swimmers, triathletes, and controls $(p>0.057)$, with one exception: the mean force repetition error of dominant shoulder extension muscles was significantly higher in swimmers than in the controls ( $p=0.045$ ) (Figure 2).

The mean peak isometric force of shoulder IR and extension muscles did not change significantly after swimming the $4.5-\mathrm{km}$ distance in free style in aerobic regimen among swimmers and triathletes (Table 3). The differences between the same muscle peak forces in swimmers and triathletes were not statistically significant $(p>0.580)$.

Despite non-significant changes of the peak isometric forces of shoulder IR and extension muscles in the dominant and non-dominant arm of swimmers and triathletes before and after the swimming trial, a significant muscle peak force side asymmetry was observed in both groups of muscles in the 2 groups of athletes after swimming the $4.5-\mathrm{km}$ distance in free style in aerobic regimen (Table 3).

The mean submaximal force reproduction error of shoulder IR and extension muscles did not change significantly after the swimming trial in swimmers and triathletes. The submaximal force reproduction error varied from $8.1 \pm 4.1 \mathrm{~N}$ to $11.6 \pm 5.5 \mathrm{~N}$ after warmup exercises and from $8.2 \pm 5.5 \mathrm{~N}$ to $15.7 \pm 10.6 \mathrm{~N}$ after swimming the $4.5-\mathrm{km}$ distance in free style in aerobic regimen. The differences between the same muscle submaximal force differentiation ability in swimmers and triathletes were not statistically significant $(p>0.230)$.

\section{Discussion}

Our swimmers and athletes trained in triathlon presented larger ROM in flexion and abduction in both arms, but IR ROM was larger only in the nondominant shoulder in comparison with the controls. The mean shoulder IR and extension muscle isometric peak forces were higher in both groups of the swimming athletes than in the controls; strength side asymmetries of these muscles were not observed in any of the 3 groups of participants. IR peak force was higher in the dominant than in the non-dominant shoulder only in the triathletes. The mean submaximal force reproduction error did not differ among participants from the 3 groups.

Shoulder IR and extension muscle peak forces did not change significantly after swimming the $4.5-\mathrm{km}$ distance in free style in aerobic regimen, but significant IR and extension muscle peak force side asymmetry appeared with stronger muscles in the dominant shoulder than in the non-dominant one. Muscle submaximal force reproduction error did not significantly increase after the swimming trial.

Our hypothesis was partly confirmed. The swimming athletes had larger shoulder ROM in flexion and abduction in both arms, as well as in IR in the non-dominant arm, but not in ER or extension. Their isometric peak forces of shoulder IR and extension muscles were significantly higher, but the force reproduction errors were not smaller in comparison with the controls. Swimming the $4.5-\mathrm{km}$ distance in free style in aerobic regimen did not deteriorate the peak forces or force differentiation ability in the swimming athletes. 
L. Irmane, I. Pontaga, I. Upitis, J. Solovjova, Swimmers' shoulder side asymmetry

The demanding training programs (including swimming, strength, and dry-land conditioning) predisposed the swimmers' shoulders to adaptive changes, including decreased IR and horizontal adduction ROM, and excessive ER in comparison with non-athletes $[8,25]$. Our present results demonstrate that shoulder ER ROM did not differ significantly in both groups of the swimming athletes and participants from the control group. Harrington et al. [26] did not detect any relationships between the characteristics of the shoulder ROM and pain appearance in swimmers' shoulders. Walker et al. [27] determined that decreased $\left(<93^{\circ}\right)$ and increased $\left(\geq 100^{\circ}\right)$ active shoulder ER ROM was an important risk factor for pain development in the shoulder joint; they did not report any correlations between the active shoulder IR ROM and the risk of pain appearance in swimmers. Our athletes exhibited the optimal shoulder ER ROM $\left(95-97^{\circ}\right)$ in accordance with the observation by Walker et al. [27]. Tate et al. [28] observed a correlation between a decreased shoulder flexion, IR ROM, and painful shoulder in young female swimmers. Hill et al. [29] similarly described decreased shoulder IR ROM and increased or decreased shoulder ER ROM as risk factors for shoulder pain development in swimmers. We observed not only optimal active shoulder ER ROM in our athletes, but also large active shoulder IR ROM (82-84 ${ }^{\circ}$ ).

The mean peak force produced by shoulder IR and extension muscles was significantly higher in our swimmers and triathletes in comparison with the controls. This proves that regular training in swimming increases shoulder IR and extension muscle strength involved in propulsion force production during swimming strokes, which is in good agreement with observations of other researchers [5, 10-12]. We did not reveal any shoulder extension muscle peak force side asymmetry in the 3 groups of our participants after warm-up exercises. The peak force of IR muscles was higher in the dominant shoulder than in the non-dominant one only in the athletes trained in triathlon. Awatani et al. [24] detected shoulder muscle maximal isometric strength side asymmetry: the strength of the dominant shoulder IR muscles was $13.3 \pm 10.7 \%$ higher than in the non-dominant shoulder, but extension strength was $21.4 \pm 12.3 \%$ higher in the dominant arm. The authors established a positive correlation between shoulder muscle maximal torque and a 25-m distance front crawl power. The correlation with swimming power was close for IR torque of the dominant (correlation coefficient $r=0.85)$ and non-dominant shoulder $(r=$ $0.76)$ and extension torque of both arms $(r=0.61)$. We could suggest from these observations [24] that the shoulder muscle strength side asymmetry was an advantage in swimmers of sprint distances, which was proved by Tourny-Chollet et al. [15]. Our swimmers and triathlon athletes swam not only short, but also longer distances. Therefore, they needed more symmetrical strength of the shoulders to avoid fast fatigue development.

Differences in force production were compared in water (12 elite swimmers performed a 30 -second tethered swimming test) and on land (4 swimmers performed isokinetic tests in shoulder and knee extension at the angular velocity of $90 \% \mathrm{~s}$ and $300 \%$ s) [30]. The authors determined significant relationships between dry-land and swimming force production $(r: 0.62$ $0.96, p<0.05$ ). The peak force, peak torque, and average torque were symmetrical in the dominant and non-dominant arms in both environments, so the asymmetries in water could be more related to swimming technical constraints than muscle strength side asymmetry.

The mean peak forces of shoulder IR and extension muscles did not change significantly after swimming the $4.5-\mathrm{km}$ distance in free style in aerobic regimen in our athletes. Despite of this finding, the peak forces of IR and extension muscles of the dominant shoulder had a non-significant tendency to increase (owing to work-in to swimming load), but the peak forces of the same muscles of the non-dominant shoulder remained unchanged (because of the initial stage of fatigue). So, the volume and intensity of the aerobic load during the swimming trial was enough to cause the significant shoulder IR and extension muscle isometric peak force side asymmetry with stronger muscles in the dominant than in the non-dominant shoulder. We suggest that this could be a reason for an unbeneficial swimming technique alteration owing to faster starting of fatigue in the non-dominant arm muscles.

Matthews et al. [18] investigated the effect of fatigue on swimming stroke length, shoulder position sense, and shoulder IR-ER maximal isometric strength (measured by the same dynamometer [MicroFET2; Hoggan Health Industries, West Jordan, USA] as we used in our research) in young swimmers; however, the participants stood upright and pressed the dynamometer against a pillar during muscle strength tests. The authors applied a fatigue protocol of an $8 \times 100 \mathrm{~m}$ swim set with a 2-minute interval and did not observe significant strength differences between pre- and postfatigue tests in IR and ER. These results are in a good agreement with our data: the peak isometric force of shoulder IR and extension did not change significantly after the swimming trial. We also detected the appear- 
ance of significant shoulder IR and extension isometric peak force side asymmetry after swimming the $4.5-\mathrm{km}$ distance in free style in aerobic regimen in our swimmers and triathletes.

Moreover, Mathews et al. [18] observed the right shoulder ROM reposition sense worsening after the fatigue protocol of interval swimming and application of passive shoulder motion: from $4.92 \pm 4.71^{\circ}$ to $9.62 \pm$ $6.63^{\circ}$. They did not detect any correlation between joint position sense and length of the swim stroke. We measured shoulder IR and extension muscle exerted submaximal force reproduction error and reported that it did not differ significantly among the 3 groups of participants. Shoulder IR and extension muscle submaximal force differentiation ability did not significantly increase after the swimming trial. Variation of the submaximal force reproduction errors is large among our swimmers and triathletes. Rejman et al. [31] determined that national level competitive swimmers who obtained lower leg muscle force repetition error in the dry-land trials were also able to better replicate this force during real swimming. Therefore, we could indirectly imply that our swimming athletes who presented the largest shoulder IR and extension muscle submaximal force reproduction errors in the dry-land tests should have worse shoulder muscle differentiation ability in the water, too.

Therefore, swimmers' shoulder early fatigue characteristic could be the muscle peak force side asymmetry appearance before worsening of the peak force absolute values and a significant decrease in submaximal force reproduction error.

A limitation of our study is that we investigated only 2 main muscle groups involved in the pull phase of the swimming stroke. The number of participants was small because a larger number of the same qualification athletes was not available. The triathletes were significantly older in comparison with the other 2 groups of participants. Older age in adolescents could have been a reason for muscle peak force increase and shoulder ROM decrease, but these were not observed in our study. Shoulder IR and extension muscle isometric peak force and the submaximal force reproduction error assessment in static mode were not specific for swimming like dynamic motions in a different environment - in water. Other fatigue protocols must be applied to evaluate their effects on shoulder muscle strength and proprioception.

As for the practical application of the study, swimming coaches and physiotherapists must consider faster fatigue occurrence in muscles of one shoulder in comparison with the other, and suggest worse shoulder muscle force differentiation error in athletes with larger submaximal force reproduction error in dry-land tests. They could involve additional resistance exercises and proprioceptive training for the weaker shoulder and in swimmers with worse shoulder muscle force differentiation ability.

\section{Conclusions}

Swimmers and triathletes presented larger ROM in flexion and abduction in both arms, but IR ROM was larger only in the non-dominant shoulder in comparison with the controls. The mean shoulder IR and extension muscle isometric peak forces were higher in both groups of swimming athletes than in the controls; strength side asymmetries of these muscles were not observed in any of the 3 groups of participants. IR peak force was higher in the dominant than in the non-dominant shoulder only in triathletes. The mean submaximal force reproduction error did not differ among participants from the 3 groups. Free-style swimming of the $4.5-\mathrm{km}$ distance in aerobic regimen caused shoulder IR and extension muscle peak force side asymmetry without a decrease of their absolute values or a significant worsening in submaximal force reproduction error.

\section{Acknowledgements}

We thank all the swimmers and triathletes who participated in this research, as well as their coaches. The study was performed without any financial support.

\section{Disclosure statement}

No author has any financial interest or received any financial benefit from this research.

\section{Conflict of interest}

The authors state no conflict of interest.

\section{References}

1. Heinlein SA, Cosgarea AJ. Biomechanical considerations in the competitive swimmer's shoulder. Sports Health. 2010;2(6):519-525; doi: 10.1177/1941738110 377611.

2. Bak K. The practical management of swimmer's painful shoulder: etiology, diagnosis, and treatment. Clin J Sport Med. 2010;20(5):386-390; doi: 10.1097/JSM. 0b013e3181f205fa.

3. Wanivenhaus F, Fox AJS, Chaudhury S, Rodeo SA. Epidemiology of injuries and prevention strategies in competitive swimmers. Sports Health. 2012;4(3):246-251; doi: 10.1177/1941738112442132. 
L. Irmane, I. Pontaga, I. Upitis, J. Solovjova, Swimmers' shoulder side asymmetry

4. Cheung ATH, Ma AWW, Fong SSM, Chung LMY, Bae Y-H, Liu KPY, et al. A comparison of shoulder muscular performance and lean mass between elite and recreational swimmers: implications for talent identification and development. Medicine. 2018;97(47):e13258; doi: 10.1097/MD.0000000000013258.

5. Habechian FAP, Malderen KV, Camargo PR, Cools AM. Changes in shoulder girdle strength in 3 consecutive years in elite adolescent swimmers: a longitudinal cohort study. Braz J Phys Ther. 2018;22(3):238-247; doi: 10.1016/j.bjpt.2018.01.001.

6. Gola R, Urbanik C, Iwańska D, Madej A. Relationship between muscle strength and front crawl swimming velocity. Hum Mov. 2014;15(2):110-115; doi: 10.2478/ humo-2014-0011.

7. Weldon EJ $3^{\text {rd }}$, Richardson AB. Upper extremity overuse injuries in swimming: a discussion of swimmer's shoulder. Clin Sports Med. 2001;20(3):423-438; doi: 10.1016/s0278-5919(05)70260-x.

8. De Martino I, Rodeo SA. The swimmer's shoulder: multi-directional instability. Curr Rev Musculoskelet Med. 2018;11(2):167-171; doi: 10.1007/s12178-0189485-0.

9. Rodeo SA, Nguyen JT, Cavanaugh JT, Patel Y, Adler RS. Clinical and ultrasonographic evaluations of the shoulders of elite swimmers. Am J Sports Med. 2016;44(12): 3214-3221; doi: 10.1177/0363546516657823.

10. Prazeres Batalha NM, de Mendonça Raimundo AM, Tomas-Carus P, Semedo Mendes Fernandes OJ, Almeida Marinho D, Rocha Martins da Silva AJ. Shoulder rotator isokinetic strength profile in young swimmers. Rev Bras Cineantropom Desempenho Hum. 2012;14(5): 545-553; doi: 10.5007/1980-0037.2012v14n5p545.

11. Olivier N, Quintin G, Rogez J. The high level swimmer articular shoulder complex [in French]. Ann Readapt Med Phys. 2008;51(5):342-347; doi: 10.1016/j.annrmp.2008.04.005.

12. Batalha N, Marmeleira J, Garrido N, Silva AJ. Does a water-training macrocycle really create imbalances in swimmers' shoulder rotator muscles? Eur J Sport Sci. 2015;15(2):167-172; doi: 10.1080/17461391.2014.908 957.

13. Psycharakis SG, Sanders RH. Shoulder and hip roll changes during 200-m front crawl swimming. Med Sci Sports Exerc. 2008;40(12):2129-2136; doi: 10.1249/ MSS.0b013e31818160bc.

14. Barden JM, Kell RT, Kobsar D. The effect of critical speed and exercise intensity on stroke phase duration and bilateral asymmetry in 200-m front crawl swimming. J Sports Sci. 2011;29(5):517-526; doi: 10.1080/ 02640414.2010.543912.

15. Tourny-Chollet C, Seifert L, Chollet D. Effect of force symmetry on coordination in crawl. Int J Sports Med. 2009;30(3):182-187; doi: 10.1055/s-0028-1104581.

16. Figueiredo P, Seifert L, Vilas-Boas JP, Fernandes RJ. Individual profiles of spatio-temporal coordination in high intensity swimming. Hum Mov Sci. 2012;31(5): 1200-1212; doi: 10.1016/j.humov.2012.01.006.
17. Higson E, Herrington L, Butler C, Horsley I. The shortterm effect of swimming training load on shoulder rotational range of motion, shoulder joint position sense and pectoralis minor length. Shoulder Elbow. 2018;10(4): 285-291; doi: 10.1177/1758573218773539.

18. Matthews MJ, Green D, Matthews H, Swanwick E. The effects of swimming fatigue on shoulder strength, range of motion, joint control, and performance in swimmers. Phys Ther Sport. 2017;23:118-122; doi: 10.1016/j. ptsp.2016.08.011.

19. Maenhout AG, Palmans T, De Muynck M, De Wilde LF, Cools AM. The impact of rotator cuff tendinopathy on proprioception, measuring force sensation. J Shoulder Elbow Surg. 2012;21(8):1080-1086; doi: 10.1016/j.jse. 2011.07.006.

20. Blonna D, Zarkadas PC, Fitzsimmons JS, O’Driscoll SW. Validation of a photography-based goniometry method for measuring joint range of motion. J Shoulder Elbow Surg. 2012;21(1):29-35; doi: 10.1016/j.jse.2011.06.018.

21. Clements AS, Ginn KA, Henley EC. Comparison of upper limb musculoskeletal function and throwing performance in adolescent baseball players and matched controls. Phys Ther Sport. 2001;2(1):4-14; doi: 10.1054/ ptsp.2000.0039.

22. Awatani T, Mori S, Shinohara J, Koshiba H, Nariai M, Tatsumi Y, et al. Same-session and between-day intrarater reliability of hand-held dynamometer measurements of isometric shoulder extensor strength. J Phys Ther Sci. 2016;28(3):936-939; doi: 10.1589/jpts.28.936.

23. Awatani T, Morikita I, Shinohara J, Mori S, Nariai M, Tatsumi Y, et al. Intra- and inter-rater reliability of isometric shoulder extensor and internal rotator strength measurements performed using a hand-held dynamometer. J Phys Ther Sci. 2016;28(11):3054-3059; doi: 10.1589/jpts.28.3054.

24. Awatani T, Morikita I, Mori S, Shinohara J, Tatsumi Y. Relationship between isometric shoulder strength and arms-only swimming power among male collegiate swimmers: study of valid clinical assessment methods. J Phys Ther Sci. 2018;30(4):490-495; doi: 10.1589/ jpts.30.490.

25. Hibberd EE, Laudner K, Berkoff DJ, Kucera KL, Yu B, Myers JB. Comparison of upper extremity physical characteristics between adolescent competitive swimmers and nonoverhead athletes. J Athl Train. 2016; 51(1):65-69; doi: 10.4085/1062-6050-51.2.04.

26. Harrington S, Meisel C, Tate A. A cross-sectional study examining shoulder pain and disability in Division I female swimmers. J Sport Rehabil. 2014;23(1):65-75; doi: 10.1123/jsr.2012-0123.

27. Walker H, Gabbe B, Wajswelner H, Blanch P, Bennell K. Shoulder pain in swimmers: a 12-month prospective cohort study of incidence and risk factors. Phys Ther Sport. 2012;13(4):243-249; doi: 10.1016/j.ptsp.2012. 01.001.

28. Tate A, Turner GN, Knab SE, Jorgensen C, Strittmatter A, Michener LA. Risk factors associated with shoulder pain and disability across the lifespan of competi- 
tive swimmers. J Athl Train. 2012;47(2):149-158; doi: 10.4085/1062-6050-47.2.149.

29. Hill L, Collins M, Posthumus M. Risk factors for shoulder pain and injury in swimmers: a critical systematic review. Phys Sportsmed. 2015;43(4):412-420; doi: 10.1080/00913847.2015.1077097.

30. Carvalho DD, Soares S, Zacca R, Marinho DA, Silva AJ, Pyne DB, et al. In-water and on-land swimmers' symmetry and force production. Int J Environ Res Public Health. 2019;16(24):5018; doi: 10.3390/ijerph16245 018.

31. Rejman M, Klarowicz A, Zatoń K. An evaluation of kinesthetic differentiation ability in monofin swimmers. Hum Mov. 2012;13(1):8-15; doi: 10.2478/v10038011-0048-0. 